\title{
Analysis of Macular Blood Flow Alterations In Thyroid Associated Ophthalmopathy
}

\author{
Xiaohan Zhang \\ Ningde Hospital affiliated to Ningde Normal College \\ Wangyuan Liu \\ Zhongshan Hospital of Fudan University \\ Zhaode Zhang \\ Ningde Hospital affiliated to Ningde Normal College \\ Jinhui Dai \\ Zhongshan Hospital of Fudan University \\ Jinfeng Zhang \\ Ningde Hospital affiliated to Ningde Normal College \\ Ling Lin ( $\square$ linling1987@outlook.com ) \\ Ningde Hospital affiliated to Ningde Normal College
}

\section{Research Article}

Keywords: Thyroid associated ophthalmopathy, Vessel density, Foveal avascular zone, Optical coherence tomography angiography

Posted Date: November 30th, 2021

DOI: https://doi.org/10.21203/rs.3.rs-1069385/v1

License: (c) (1) This work is licensed under a Creative Commons Attribution 4.0 International License. Read Full License 


\section{Abstract}

Purpose: To evaluate the alterations in macular superficial retinal vessels density and relation with visual acuity in thyroid associated ophthalmopathy (TAO) patients with different severity levels.

Method: This cross-sectional observational study included 70 TAO patients and 70 healthy controls. Only the data from the right eyes were analyzed. TAO patients were divided into 7 subgroups according to the NOSPECS score grading from 0 to 6 with 10 cases in each subgroup. Foveal avascular zone (FAZ), vascular density (VD) and perfusion density (PD) of macular $1 \mathrm{~mm}$ diameter and $6 \mathrm{~mm}$ diameter areas were measured by optical coherence tomography angiography (OCTA).

Results: In TAO patients, significant increases were found in macular and foveal vascular densities (FVD) and perfusion densities (FPD) while significant decrease was found in FAZ area when comparing with control group $(\mathrm{p}<0.05)$. Spearman correlation analysis and multiple linear regression analysis showed that TAO severity grade was negatively correlated with FVD $(\beta=-1.150, p=0.032), \operatorname{FPD}(\beta=-0.024, p=0.042), M V D(\beta=-0.583, p=0.020)$ and MPD $(\beta=-0.011$, $p=0.010)$. Clinical activity score (CAS) score showed positive correlation with $F V D(\beta=0.794, p=0.035)$ and $F P D(\beta=0.017, p=0.041)$. $F V D(\beta=-0.009, p=0.033)$, $\operatorname{MVD}(\beta=-0.034, p<0.001), \operatorname{FPD}(\beta=-0.416, p=0.039)$ and $\operatorname{MPD}(\beta=-2.428, p<0.001)$ all showed negative correlation with best corrected visual acuity $(B C V A)$.

Conclusion: Superficial macular blood flow in TAO patients showed an overall increase comparing with healthy controls and a decrease as TAO disease severity worsen. Superficial macular blood flow density was negatively correlated with BCVA.

\section{Background}

Thyroid associated ophthalmopathy (TAO) is an orbital disease that accompanied with abnormal thyroid endocrine axis dysfunction. Though the cause of the disease has not yet been fully revealed, TAO is now recognized as a systemic autoimmune disorder or an organ-specific autoimmune inflammatory disease, which also closely related to the function of systemic circulatory system [1-3]. Meanwhile, retina, which could reflect the microvascular circulation of different vascular beds, is a window for the observation of systemic microvascular function. Li H [4] found that the blood flow of the central retinal artery positively correlated with the blood flow of the superior thyroid artery in TAO patients using color Doppler (CDFI), which could be used to evaluate the treatment of hyperthyroid exophthalmia. Up to now, many techniques have been used to investigate the blood flow of retina and choroid in TAO, such as Laser Doppler flow meter, indocyanine green angiography and laser speckle imaging. However, the non-quantitative and invasive characteristics of these techniques set limitations for the observation of retinal microcirculation. As an important extension of optical coherence tomography (OCT), optical coherence tomography angiography (OCTA) is used to further observe the blood flow of retina and choroid clinically. It shows good repeatability and reproducibility in the quantitative analysis of retinal blood flow in normal eye-based optical microangiography (OMAG) [5]. Thus, as a new type of non-invasive imaging technique, OCTA has great potential for evaluation of blood flow in the retina and choroid, in both scientific research and clinical practice.

OMAG provides numerous quantitative parameters to analyze retinal blood flow, such as vessel density (VD) and perfusion density (PD). VD considers only the length of the blood flow signal which is conducive to display the perfusion in capillary level, while PD considers both the length and width of the blood flow signal. Recently, OCTA has been used to diagnose and quantify changes in retinal blood flow, such as quantifying the macular density in patients with diabetic retinopathy, branch vein occlusion or glaucoma, and even have been used to assess the impact of systemic diseases on retinal blood flow [6-8]. Ye [9] reported the use of OCTA to evaluate the retinal microcirculations in active TAO patients, which showed that the macular microcirculations density changed in both the superficial and deep retina. However, this study did not compare the difference of retinal microcirculations in patients with different severity grades of TAO.

In this study, we use OCTA to evaluate changes in superficial macular blood flow density and FAZ of patients with different severity grades of TAO. Furthermore, we aim to explore the influence of TAO on the superficial retinal microcirculation and the correlation between macular blood flow of TAO and visual acuity.

\section{Methods Subjects}

This cross-sectional observational study enrolled 70 untreated TAO patients who were diagnosed in the Ophthalmology and Endocrinology departments in Ningde Hospital (Fujian, China) from June 2017 to June 2019 (TAO group). The protocol of the study was approved by the Scientific and Ethics Committees of Ningde Hospital affiliated to Ningde Normal College and was conducted in accordance with the tenets of the Declaration of Helsinki. Informed consent was obtained from all the patients in this study.

70 participants without systematic or ocular diseases were included in the control group (HC group). Only the right eyes were analyzed in this study. Al patients underwent clinical examination and assessment processed by Ophthalmologist and Endocrinologist including best corrected visual acuity(BCVA), intraocular, refraction, eyelid aperture, ocular protrusion, extra-ocular muscle thickness measured by B ultrasound, cornea evaluation, fundus examination and clinical activity score (CAS) evaluation [10]. TAO diagnosis was according to the standard of Barley and Gorman [11]. TAO patients were graded from 0 to 6 according to the NOSPECS classification [12] and 10 patients were enrolled in each grade. Exclusion criteria included: (1) circulatory and metabolic diseases including diabetes, hypertension, and heart disease; (2) ocular diseases besides TAO; (3) smoking and alcoholism; (4) long-term medication history; (5) Pregnant and lactating female; (6) History of eye surgery.

\section{OCTA}


OCTA was performed in all subjects. A Cirrus HD-OCT5000(Carl Zeiss, Germany) was used to scan the macular area with a scanning range of 6 mm $\times 6$ mm. The subject was asked to place the mandible and forehead on the chin rest and forehead rest respectively, and stared at the green fixation cursor from the lens for more than 3 seconds for scanning. FastTrac@ image tracking function was turned on for all scans. OCTA image quality quantitative evaluation was adopted and images with a quality of $<8$ were not included in the study. OCTA data was automatically imported into the FORUM system (version 4.0) and was quantitatively analyzed by its own analysis software (angio-analytics). Superficial retinal layer (SRL) was calculated from the internal limiting membrane (ILM) to the inner plexiform layer (IPL). A $6 \times 6 \mathrm{~mm}$ macular scan was used to measure superficial macular capillary plexus. Macular area was divided into the central area, inner ring, outer ring and intact area according to Early Treatment Diabetic Retinopathy Study (ETDRS) to quantitatively analyze PD and VD (Figure 1). Because the central and intact areas could better represent the macular blood flow, we only analyzed central area and intact area in this study, which are the foveal vessel density (FVD) and foveal perfusion density (FPD) of the $1 \mathrm{~mm}$ diameter fovea and macular vessel density (MVD) and macular perfusion density (MPD) of the $6 \mathrm{~mm}$ diameter macula. FAZ was manually checked before the software automatically calculates its area. All examinations were completed by the same physician and read by two physicians independently. If the two physicians disagree, a third physician will decide.

\section{Statistical Analysis}

All data were expressed as means \pm standard deviations and were analyzed with SPSS software (version 25.0; SPSS, Inc., Chicago, IL, USA). Chi-square analysis was used for categorical data. The influence of age, eyelid aperture, IOP, TAO grade, and CAS on FAZ and macular blood flow in TAO group were analyzed by Spearman correlation test and multiple linear regression analysis. The effects of FAZ and retinal blood flow on BCVA in the TAO group were analyzed by linear regression. The differences in FAZ and retinal blood flow between subjects (control group and TAO subgroups) were analyzed by KruskalWallis rank test. A P value of $<0.05$ was considered statistically significant.

\section{Result}

In total, 140 participants, comprising 70 TAO patients (70 eyes) and 70 healthy controls (70 eyes) were included in this study. No significant difference was found in age, gender and IOP between TAO and control groups, while significant differences were found in BCVA, eyelid aperture distance and CAS (Table 1).

Table 1. Demographics and clinical characteristics of the subjects.

\begin{tabular}{|c|c|c|c|}
\hline & $\mathrm{TAO}(\mathrm{n}=70)$ & $\mathrm{HC}(\mathrm{n}=70)$ & $\mathrm{P}$ \\
\hline Age (mean \pm SD $\llbracket$ year) & $39.7 \pm 9.9$ & $42.20 \pm 10.8$ & $0.151^{*}$ \\
\hline Sex (male/female) & $26 / 44$ & $30 / 40$ & $0.605+$ \\
\hline IOP $(m e a n \pm S D \llbracket m m H g)$ & $16.6 \pm 3.0$ & $15.9 \pm 1.6$ & $0.086^{*}$ \\
\hline BCVA (mean \pm SD, Log MAR) & $0.06 \pm 0.14$ & $0.02 \pm 0.03$ & $0.025^{\star}$ \\
\hline Eyelid aperture distance (mm) & $14.7 \pm 1.7$ & $12.0 \pm 0.7$ & $\otimes 0.001^{\star}$ \\
\hline CAS & $3.0 \pm 2$ & $1.2 \pm 0.9$ & $\otimes 0.001^{\star}$ \\
\hline
\end{tabular}

$\mathrm{IOP}=$ Intraocular pressure, BCVA=Best-corrected visual acuity; CAS=Clinical activity score. *Student's t test, + Chi-squared test.

\section{Differences in FAZ and macular blood flow between TAO and control groups}

In TAO group, FAZ was found significantly decreased comparing with control group. While all the macular blood flow parameters in TAO (FVD, MVD, FPD and $<$ PD), was significantly increased (Table 2).

Table 2. FAZ and macular blood flow differences between TAO and control groups

\begin{tabular}{|c|c|c|c|c|c|}
\hline & FAZ & FVD & MVD & FPD & MPD \\
\hline TAO & $0.22(0.14 \sim 0.30)$ & 11.8(7.6 13.7) & 18.5(18.0 19.0) & $0.271(0.181 \sim 0.314)$ & $0.459(0.446 \sim 0.466)$ \\
\hline $\mathrm{HC}$ & $0.34(0.29 \sim 0.43)$ & 7.3(4.9 9.0) & 17.7(17.3 18.2) & $0.164(0.109 \sim 0.192)$ & $0.439(0.426 \sim 0.452)$ \\
\hline Z & -6.446 & -5.705 & -4.617 & -6.124 & -4.597 \\
\hline $\mathrm{p}$ & $\nabla 0.001$ & 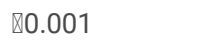 & $\triangle 0.001$ & $\otimes 0.001$ & $\otimes 0.001$ \\
\hline
\end{tabular}

$F A Z=$ foveal avascular zone; FVD= foveal vessel density; MVD=macular vessel density; FPD=foveal perfusion density; MPD=macular perfusion density; F= degrees of freedom in Kruskal-Wallis $\mathrm{H}$ test (control group and 7 TAO subgroups)

\section{Differences in FAZ, BCVA and macular blood flow between TAO subgroups and control group}

Table 3. FAZ, BCVA and macular blood flow differences between TAO subgroups and control group 


\begin{tabular}{|lllllll|}
\hline & FAZ & FVD & MVD & FPD & MPD & BCVA aLogMAR口 \\
\hline Kruskal-Wallis H(K) & 54.596 & 45.141 & 38.593 & 50.005 & 35.113 & 52.057 \\
\hline F & 7 & 7 & 7 & 7 & 7 & 7 \\
\hline P & $\mathbb{0 0 . 0 0 1}$ & $\square 0.001$ & $\square 0.001$ & $\square 0.001$ & $\square 0.001$ & $\square 0.001$ \\
\hline
\end{tabular}

$F A Z=$ foveal avascular zone; $F V D=$ foveal vessel density; $M V D=$ macular vessel density; $F P D=$ foveal perfusion density; $M P D=$ macular perfusion density; $B C V A=$ best corrected visual acuity; $\mathrm{F}=$ degrees of freedom in Kruskal-Wallis $\mathrm{H}$ test (control group and 7 TAO subgroups)

Kruskal-Wallis $\mathrm{H}$ test showed that there were significant differences among 7 TAO subgroups and control group in FAZ, FVD, MVD, FPD, MPD and BCVA (Table 3). Further intra-group analysis showed that significant decrease was found in FAZ in TAO grade0, grade1, grade4 and grade6 comparing to control group $(p=0.011,<0.001,<0.001,<0.001)$. Moreover, significant increase was found in FVD and FPD in TAO grade0, grade1, and grade4 comparing to control group $(p=0.005,0.001,<0.001 ; p=0.003,0.001,<0.001)$, while only TAO grade0 and grade1 showed increase in MVD and MPD comparing to control group ( $p=0.016$, $<0.001 ; p=0.011,<0.001)$. TAO grade6 showed a significant decrease in MVD comparing to TA01 ( $p=0.007)$. However, grade6 showed the best BCVA than all the other 7groups (all $p<0.001$ ) (Fig 2).

3. Correlation between ocular parameters and macular blood flow

Table 4. Correlation between ocular parameters and macular blood flow

\begin{tabular}{|c|c|c|c|c|c|c|c|c|c|c|c|c|c|c|c|}
\hline \multirow{2}{*}{$\begin{array}{l}\text { Dependent } \\
\text { variable }\end{array}$} & \multicolumn{3}{|c|}{ TAO grade } & \multicolumn{3}{|l|}{ CAS } & \multicolumn{3}{|c|}{ Age(year) } & \multicolumn{3}{|c|}{ IOPImmHg] } & \multicolumn{3}{|c|}{$\begin{array}{l}\text { Eyelid aperture distance } \\
\text { [mm! }\end{array}$} \\
\hline & B & Beta & $\begin{array}{l}\mathrm{P} \text { - } \\
\text { value }\end{array}$ & B & Beta & $\begin{array}{l}\mathrm{P} \text { - } \\
\text { value }\end{array}$ & B & Beta & $\begin{array}{l}\mathrm{P} \text { - } \\
\text { value }\end{array}$ & B & Beta & $\begin{array}{l}\mathrm{P}- \\
\text { value }\end{array}$ & B & Beta & $\begin{array}{l}\mathrm{P} \text { - } \\
\text { value }\end{array}$ \\
\hline $\mathrm{FAZ} \square \mathrm{mm}^{2} \square$ & 0.016 & 0.246 & 0.437 & -0.009 & -0.156 & 0.506 & 0.003 & 0.210 & 0.138 & -0.006 & -0.135 & 0.481 & -0.004 & -0.058 & 0.740 \\
\hline $\begin{array}{l}\text { FVD }\left(\mathrm{mm}^{-}\right. \\
\left.{ }^{1}\right)\end{array}$ & -1.150 & -0.618 & 0.032 & 0.794 & 0.449 & 0.035 & -0.114 & -0.300 & 0.019 & 0.147 & 0.118 & 0.492 & 0.239 & 0.106 & 0.494 \\
\hline $\begin{array}{l}\text { MVD } \\
\left(\mathrm{mm}^{-}\right)\end{array}$ & -0.583 & -0.695 & 0.020 & 0.340 & 0.426 & 0.053 & -0.033 & -0.191 & 0.146 & 0.059 & 0.105 & 0.555 & 0.088 & 0.087 & 0.588 \\
\hline FPD & -0.024 & -0.599 & 0.042 & 0.017 & 0.445 & 0.041 & -0.002 & -0.259 & 0.048 & 0.003 & 0.124 & 0.482 & 0.003 & 0.072 & 0.649 \\
\hline MPD & -0.011 & -0.748 & 0.010 & 0.006 & 0.413 & 0.052 & -0.010 & -0.207 & 0.103 & 0.001 & 0.054 & 0.753 & 0.002 & 0.111 & 0.477 \\
\hline
\end{tabular}

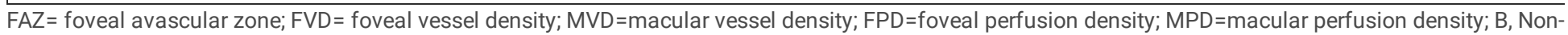
standardized coefficient; Beta, B standardized coefficient. Boldface numbers indicate statistically significant differences at $\mathrm{p}<0.05$.

Age, palpebral fissure height, IOP, TAO severity grade and CAS score were taken as independent variables in spearman correlation analysis and multiple linear regression analysis (Table 4). The analysis showed that TAO severity grade, CAS score, age, palpebral fissure height and IOP are not influencing factors of FAZ in TAO patients. TAO classification and age are negatively correlated with FVD and FPD in TAO patients, while CAS score is positively correlated with FVD and FPD in TAO patients. The palpebral fissure height and IOP are not independent influencing factors of FVD and FPD in TAO patients. TAO classification is negatively correlated with MVD and MPD in TAO patients. CAS score, age, palpebral fissure height and IOP are not independent influencing factors of MVD and MPD in TAO patients.

\section{Correlation between macular blood flow and BCVA in TAO patients}

Negative correlation was found between BCVA (log MAR) and FVD, MVD, FPD and MPD, which indicates that the BCVA decreases with the blood flow density decreasing in $1 \mathrm{~mm}$ diameter and $6 \mathrm{~mm}$ diameter macular areas. No significant correlation was found between BCVA and FAZ (Table 5).

Table 5. Influence of FAZ and macular blood blow on BCVA(LogMAR) in TAO patients

\begin{tabular}{|c|c|c|c|c|c|c|}
\hline & B & Std.Error & Beta & $t$ & $P$ & $\mathrm{R}^{2}$ \\
\hline FAZ & -0.035 & 0.129 & -0.033 & -0.272 & 0.786 & 0.001 \\
\hline FVD & -0.009 & 0.004 & -0.255 & -2.176 & 0.033 & 0.065 \\
\hline MVD & -0.034 & 0.009 & -0.425 & -3.874 & {$[0.001$} & 0.181 \\
\hline FPD & -0.416 & 0.198 & -0.247 & -2.103 & 0.039 & 0.061 \\
\hline MPD & -2.428 & 0.495 & -0.511 & -4.907 & {$[0.001$} & 0.261 \\
\hline
\end{tabular}

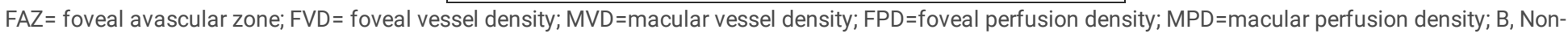
standardized coefficient; Beta, B standardized coefficient. Boldface numbers indicate statistically significant differences at $\mathrm{p}<0.05$. 


\section{Discussion}

TAO is one of the complications of hyperthyroidism that mainly involve the inflammation and tissue expansion of the orbit. In the past, many efforts had been devoted to finding indicators for evaluating activity of TAO disease quantitively. However, people can only organize the tissues based on the soft tissue edema of the eye, vasodilatation, Computed Tomography (CT) and Magnetic Resonance Imaging (MRI) imaging. Nowadays, OCTA is a fast, convenient, non-invasive, and easy to quantitatively measure the blood flow in the macular area, which also provides a way to evaluate and further predict the severity of TAO disease. In this study, we used OCTA to measure the macular retinal blood flow and FAZ area in TAO patients. Comparing with control group, TAO group showed significant decrease in FAZ area and increase in FVD, FPD, MVD and MPD. In order to further explore the relationship between the severity of TAO and the macular blood flow, we further subdivided the TAO group according to NOSPECS score to investigate the changes in the retinal microcirculation and FAZ area in TAO patients.

The blood flow change in TAO is associated with both systemic and ocular factors. Hyperthyroidism increased systemic pressure and cardiac output which may enhance orbital blood flow [13]. Mehmet also observed an increase of systolic blood flow velocity in central retinal blood flow in TAO patients using color Doppler ultrasound. Moreover, Kurioka [14] found that the orbital inflammatory changes in TAO also causes the increase of blood flow velocity in most of the TAO patients. However, in the late stage of TAO, degeneration and fibrosis of orbital muscles and fat tissues lead to the decrease of retinal blood flow density. Previous studies on TAO macular blood flows using OCTA showed that superficial vessel density was lower in active TAO and higher in inactive TAO comparing with normal controls [15-18]. In our study, we observed an overall increase of superficial vessel density in TAO patients comparing to controls. We further classified TAO patients based on NOSPEC score. Our analysis showed that as the severity of TAO increased, the $1 \mathrm{~mm}$ and $6 \mathrm{~mm}$ diameter macular vessel densities decreased. Further subgroup comparisons also revealed that comparing to control group, only TAO grade0 and grade1 showed significant increase in superficial vessel density. Our results suggested that macular blood flow density increased significantly in the early state of the disease and then gradually decreased and stabilized. According to NOSPEC classification, minor TAO related ocular changes were observed in grade 0 and grade1. Therefore, with systemic increase of blood pressure and output, an increase of macular blood flow density was observed in TAO patients from grade 0 and grade1. As ocular symptoms worsen, a trend of decease in macular blood flow was observed in TAO subgroups. It should be noted that TAO grade 4 showed an increase of foveal vessel density comparing with control group while no significant increase was found in macular vessel density. Previous study from Perri [19] reported that extraocular muscle hypertrophy was positively correlated with retinal blood flow. Thus, we speculated that the transient increase in foveal vessel density in TAO grade 4 was the combined effects of extraocular muscle hypertrophy and higher clinical activity. The microcirculation state of the patient's macular area is not only related to the severity of the disease, but also the inflammatory factors and extraocular muscles.

Previous researches found an enlargement of FAZ area in active TAO while no significant change in non-active TAO [16]. In our study, we found an overall decrease of FAZ area in TAO patients while further analysis showed no correlation between severity of TAO disease and FAZ area. After subgroup comparisons, the FAZ areas of TAO grade0, 1, 4 and 6 were significantly smaller than that of the control group. This result is consistent with the increase of foveal vessel density in TAO grade0, 1, 4 and 6 comparing with control group, that the central avascular area narrows with the foveal vessel density enlarges. In addition, our analysis showed no significant correlation between the FAZ area and BCVA. This finding indicated that FAZ area changing is not the cause of BCVA decline in TAO, which is different from pathological mechanism of diabetic retinopathy, retinal vein occlusion and other diseases that destroyed arch ring caused ischemia of the foveal and decreased visual acuity. However, our analysis showed that macular vessel density positively correlated with BCVA, which suggested that the TAO caused BCVA decline might be caused by macular microcirculation change. Therefore, we speculated that the changes in macular microcirculation have already affected visual function before the significant decrease in BCVA caused by optic nerve damage in TAO grade6. The macular vessel density especially foveal vessel density showed decrease in TAO grade5 while BCVA(LogMAR) started to increase, which indicated the macular microcirculation change in TAO grade5 has already have impact on visual function. We speculated that he abundant blood flow and fast metabolic rate of macular area in TAO patients also made it sensitive to hypoxia. Thus, the slightly decline of blow flow density might influence the visual function in TAO patients. Moreover, since the blood flow density in TAO grade6 was not significantly decrease comparing with TAO grade5, we considered that optic nerve damage played a decisive role in visual acuity decline. In summary, we believe that BCVA of middle and late stages TAO patients are also affected by changes in the macular microcirculation. When the TAO severity is assessed as grade 4, the macular blood flow density, especially FVD and FPD, should be monitored regularly. Once a significant decline in macular blood flow density is detected, it is necessary to be alert that whether TAO will worsen into grade 5. At this time, besides conventional treatment, additional clinical intervention to promote retinal microcirculation and protect visual function should be considered.

Our study is the first horizontal study on macular $6 \times 6 \mathrm{~mm}$ diameter OCTA parameters in TAO classified by NOSPECS score. Because shadows and projection artifacts in OCTA that have a greater impact on deep retinal blood flow, only superficial retinal blood flow parameters were analyzed. Therefore, the blood flow changes in the deep retina of TAO patients need to be further explored. Moreover, since sample size of each subgroup in this study is slightly insufficient, further cross-sectional observational study and longitudinal follow-up studies with larger sample sizes are needed to dynamically observe the changes in retinal blood flow in TAO.

\section{Conclusion}

Our study analyzed the macular superficial blood flow density and FAZ area in TAO patients with different severity degrees. Our result showed that superficial vessel density showed an overall increase in TAO patients comparing with healthy controls and a trend of decrease as TAO disease severity worsen. FAZ area showed an increase in TAO patients while this change was not correlated with TAO severity. Our study suggested that macular superficial blood flow density was negatively correlated with BCVA(LogMAR). Thus, when TAO NOSPECS grade reaches grade 5 , systemic and ocular microcirculation intervention should be considered to protect visual function.

\section{Abbreviations}


TAO: thyroid associated ophthalmopathy, FAZ: Foveal avascular zone, VD: vascular density, PD: perfusion density, OCTA: optical coherence tomography angiography, FVD: foveal vascular densities, FPD: foveal perfusion densities, CAS: clinical activity score, BCVA: best corrected visual acuity, OCT: optical coherence tomography, OMAG: normal eye-based optical microangiography, SRL: superficial retinal layer, ILM: internal limiting membrane, IPL: inner plexiform layer, EDTRS: Early Treatment Diabetic Retinopathy Study, CT: Computed Tomography, MRI: Magnetic Resonance Imaging

\section{Declarations}

Ethics approval and consent to participate: This study was approved by the Ethnical Committee of Ningde Hospital and was performed according to the principles of the Declaration of Helsinki. The method and purpose of this study were explained in detail and consent was obtained from each patient.

Consent for publication: Not applicable.

Availability of data and material: The datasets used and/ analyzed during the current study are available from the corresponding author on reasonable request.

Competing interests: The authors declare that they have no competing interests.

Funding: This work was supported by Grant 81470657 and Grant 81970831 from the National Natural Science Foundation of China, and Grant 2019 QH1307 from Qihang Foundation of Fujian Medical University. The sponsors or funding organizations had no role in the design or conduct of this research.

Authors' contributions: XHZ and WYL performed the data analyses and wrote the main manuscript text. LL contributed to the conception of the study. JFZ, ZDZ and JHD helped perform the analysis with constructive discussions. All authors reviewed the manuscript.

Acknowledgements: We do not have anyone to acknowledge.

\section{References}

1. Bahn RS. Current Insights into the Pathogenesis of Graves' Ophthalmopathy. Horm Metab Res. 2015;47(10):773-8.

2. Wang Y, Smith TJ. Current concepts in the molecular pathogenesis of thyroid-associated ophthalmopathy. Invest Ophthalmol Vis Sci. 2014; 55(3):173548.

3. Yang X, Huang D, Ai S, Liang X, Zhao J, Fang L. Retinal Vessel Oxygen Saturation and Vessel Diameter in Inactive Graves Ophthalmopathy. Ophthal Plast Reconstr Surg. 2017;33(6):459-465.

4. Li H, Liu YH, Li DH, Zhang Y. Value of measurements of blood flow velocity in central retinal artery in thyroid-associated ophthalmopathy. Zhongguo Yi Xue Ke Xue Yuan Xue Bao. 2004;26(4):460-462.

5. Lee TH, Lim HB, Nam KY, Kim K, Kim JY. Factors Affecting Repeatability of Assessment of the Retinal Microvasculature Using Optical Coherence Tomography Angiography in Healthy Subjects. Sci Rep.2019;9(1):16291.

6. Kim AY, Chu Z, Shahidzadeh A, Wang RK, Puliafito CA, Kashani, AH. Quantifying Microvascular Density and Morphology in Diabetic Retinopathy Using Spectral-Domain Optical Coherence Tomography Angiography. Invest Ophthalmol Vis Sci.2016;57(9): 362-70.

7. Shin JW, Sung KR, Lee JY, Kwon J, Seong M. Optical coherence tomography angiography vessel density mapping at various retinal layers in healthy and normal tension glaucoma eyes. Graefes Arch Clin Exp Ophthalmol.2017;255(6):1193-1202.

8. Lee WH, Park JH, Won Y, et al. Retinal Microvascular Change in Hypertension as measured by Optical Coherence Tomography Angiography. Sci Rep.2019;9(1):156.

9. Ye L, Zhou SS, Yang WL. RETINAL MICROVASCULATURE ALTERATION IN ACTIVE THYROID-ASSOCIATED OPHTHALMOPATHY. ENdocr Pract.2018;24(7):658-667.

10. Menconi F, Profilo MA, Leo M, Spontaneous improvement of untreated mild Graves 'ophthalmopathy: Rundle's curve revisited. Thyroid.2014;24:60-66.

11. Perri P, Campa C, Costagliola C, et al. Increased retinal blood flow in patients with active Graves' ophthalmopathy. Curr Eye Res. 2007;32:985-990.

12. Werner SC. Classification of the eye changes of Graves'disease. Am J Ophthalmol,1977,83(5):725-727.

13. J Merc'e, S Ferr'as, C Oltra, Cardiovascular abnormalities in hyperthyroidism: a prospective doppler echocardiographic study, American Journal of Medicine, 2005;118(2):126-131.

14. Kurioka Y, Inaba M, Kawagishi T, et al. Increased retinal blood flow in patients with Graves' disease: influence of thyroid function and ophthalmopathy. Eur J Endocrinol.2001;144(2):99-107.

15. Mansoreh Jamshidian Tehrani,Zahra Mahdizad,Abolfazl Kasaei, et al. Early macular and peripapillary vasculature dropout in active thyroid eye disease. Graefe's Archive for Clinical and Experimental Ophthalmology, 2019:257(11):2533-2540.

16. Yu L, Qin J, Yu C, et al. Evaluation of retinal and choroidal variations in thyroid-associated ophthalmopathy using optical coherence tomography angiography. BMC Ophthalmol,2020;20(1):421-425.

17. Cetin A, Muhammed MK, Merve Y, Analysis of Foveal and Parafoveal Microvascular Density and Retinal Vessel Caliber Alteration in Inactive Graves' Ophthalmopathy. Journal of Ophthalmology 2020;2(2020):1-8.

18. Fazil K, Ozturk K. G, Alkin Z. Evaluation of Choroidal Thickness and Retinal Vessel Density in Patients with Inactive Graves' Orbitopathy. Photodiagnosis and Photodynamic Therapy, 2020;32:101898.

19. Perri P, Campa C, Costagliola C, et al. Increased retinal blood flow in patients with active Graves' ophthalmopathy. Curr Eye Res. 2007;32(11):985-90.

Page 6/8 


\section{Figures}
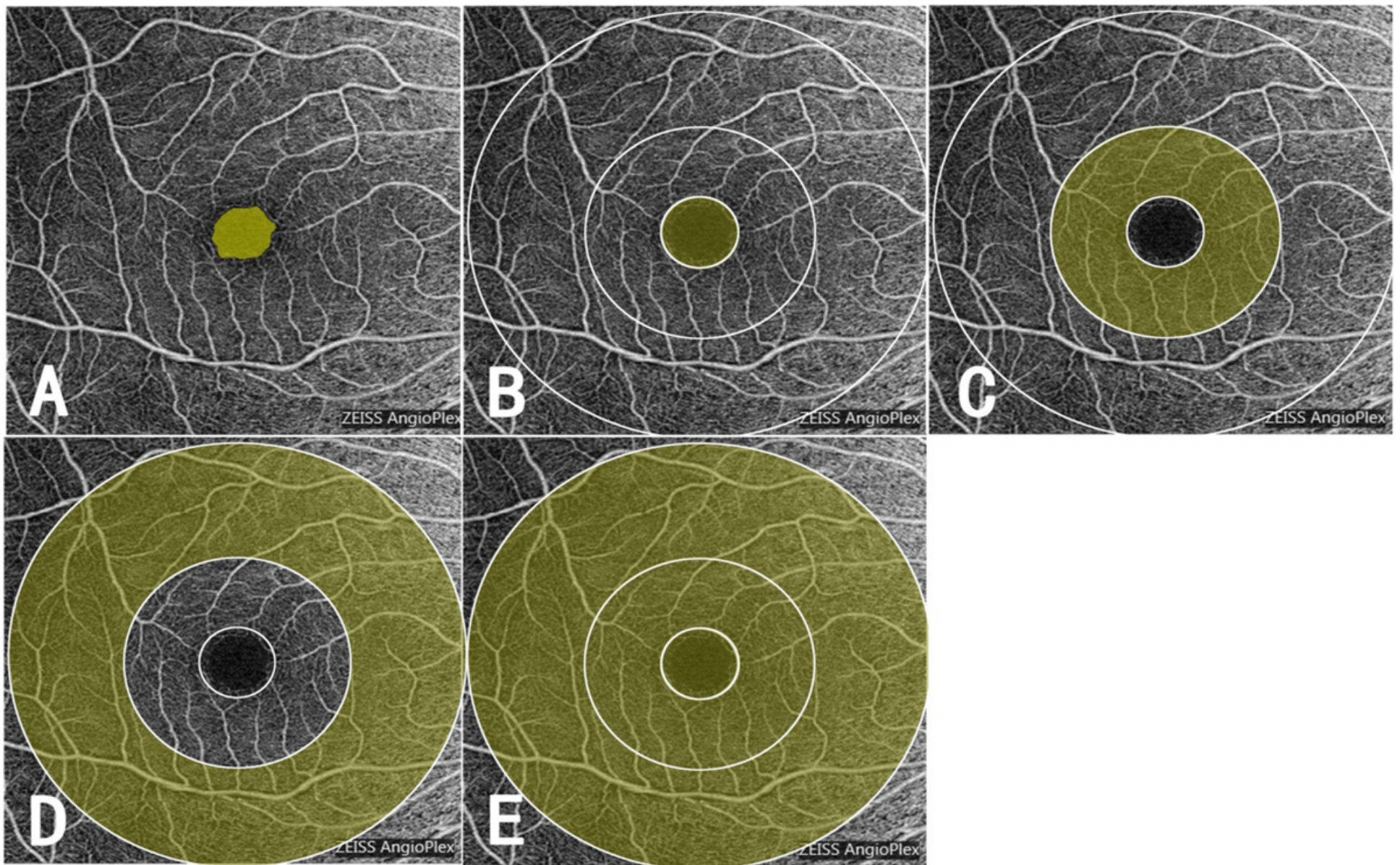

Figure 1

OCTA of the macular area. A. FAZ border after manual checking; B-E: ETDRS grid was divided into the following regions: region 1, central 1-mm (B); region 2, the inner ring (C); region 3, the outer ring (D); region 4, $6 \mathrm{~mm}$ intact circle (E). Blood flow density in region 1 was indicated by FVD and FPD; blood flow density in region 4 was represented by MVD and MPD. 

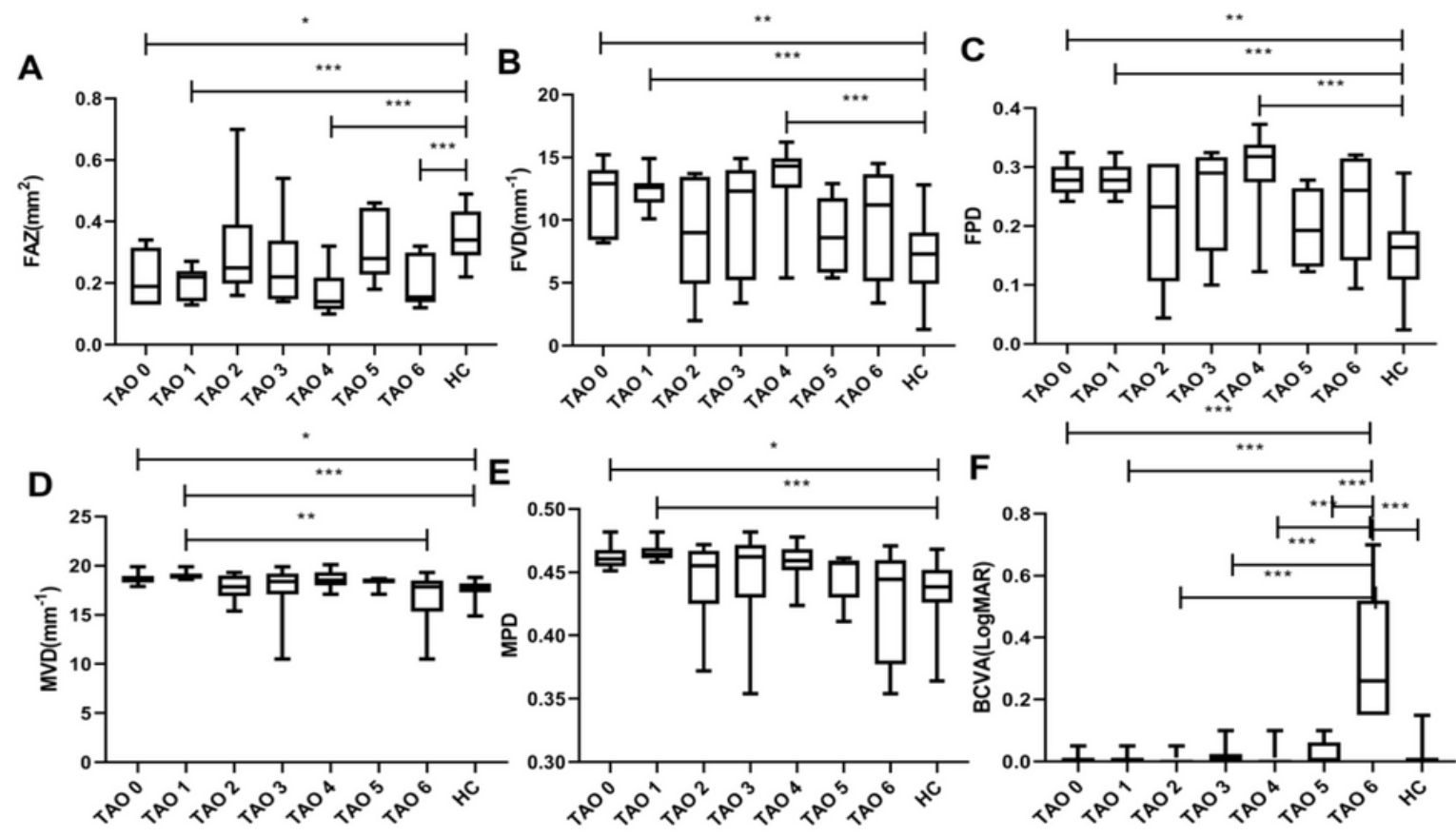

Figure 2

Intra-group comparisons in FAZ, BCVA and macular blood flow among TAO subgroups and control group. A. Intra-group comparison in FAZ. B. Intra-group comparison in FVD. C. Intra-group comparison in FPD. D. Intra-group comparison in MVD. E. Intra-group comparison in FAZ. F. Intra-group comparison in BCVA.

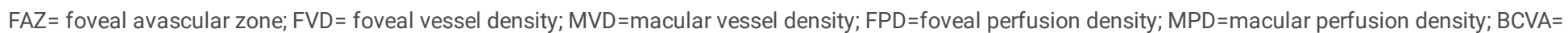
best corrected visual acuity. * $p<0.05 ;{ }^{* *} p<0.01 ; * \star * p<0.001$. 